\title{
Structural Destabilization of Azurin by Imidazolium Chloride Ionic Liquids in Aqueous Solution
}

Arusha Acharyya, ${ }^{1}$ David DiGiuseppi, ${ }^{2}$ Brittany L. Stinger, ${ }^{3}$ Reinhard Schweitzer-Stenner, ${ }^{2}$ and Timothy D. Vaden ${ }^{3 *}$

${ }^{1}$ Department of Chemistry, University of Pennsylvania, 231 S. 34 Street, Philadelphia, PA 19104

${ }^{2}$ Department of Chemistry, Drexel University, 32 S. 32nd Street, Philadelphia, PA 19104

${ }^{3}$ Department of Chemistry and Biochemistry, Rowan University, 201 Mullica Hill Road, Glassboro, NJ 08028

* To Whom Correspondence Should Be Addressed: vadent@ rowan.edu

\section{Supporting Information}

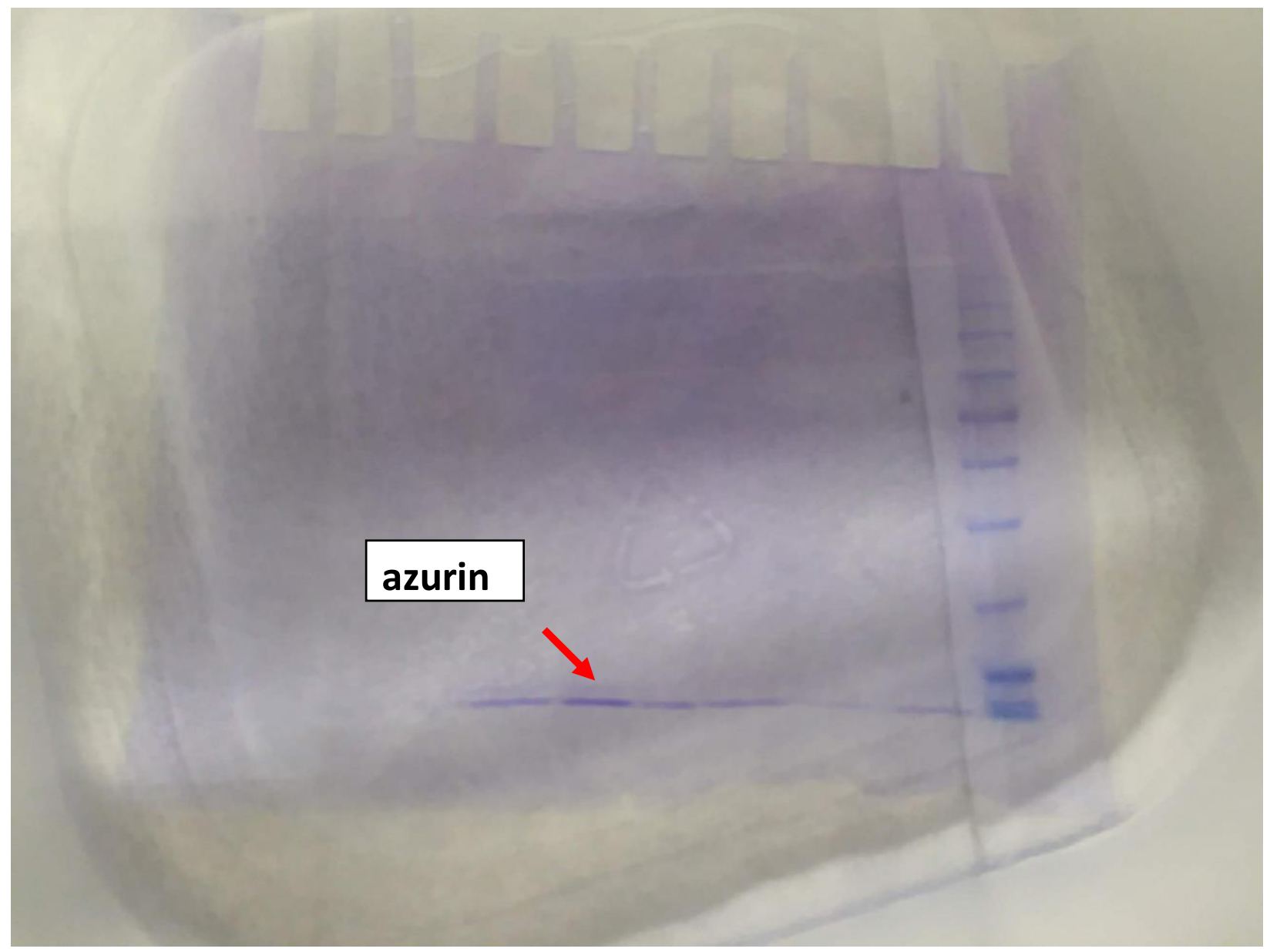

Figure S1. Gel electrophoresis results demonstrating purity of azurin expressed and extracted from E. coli. 


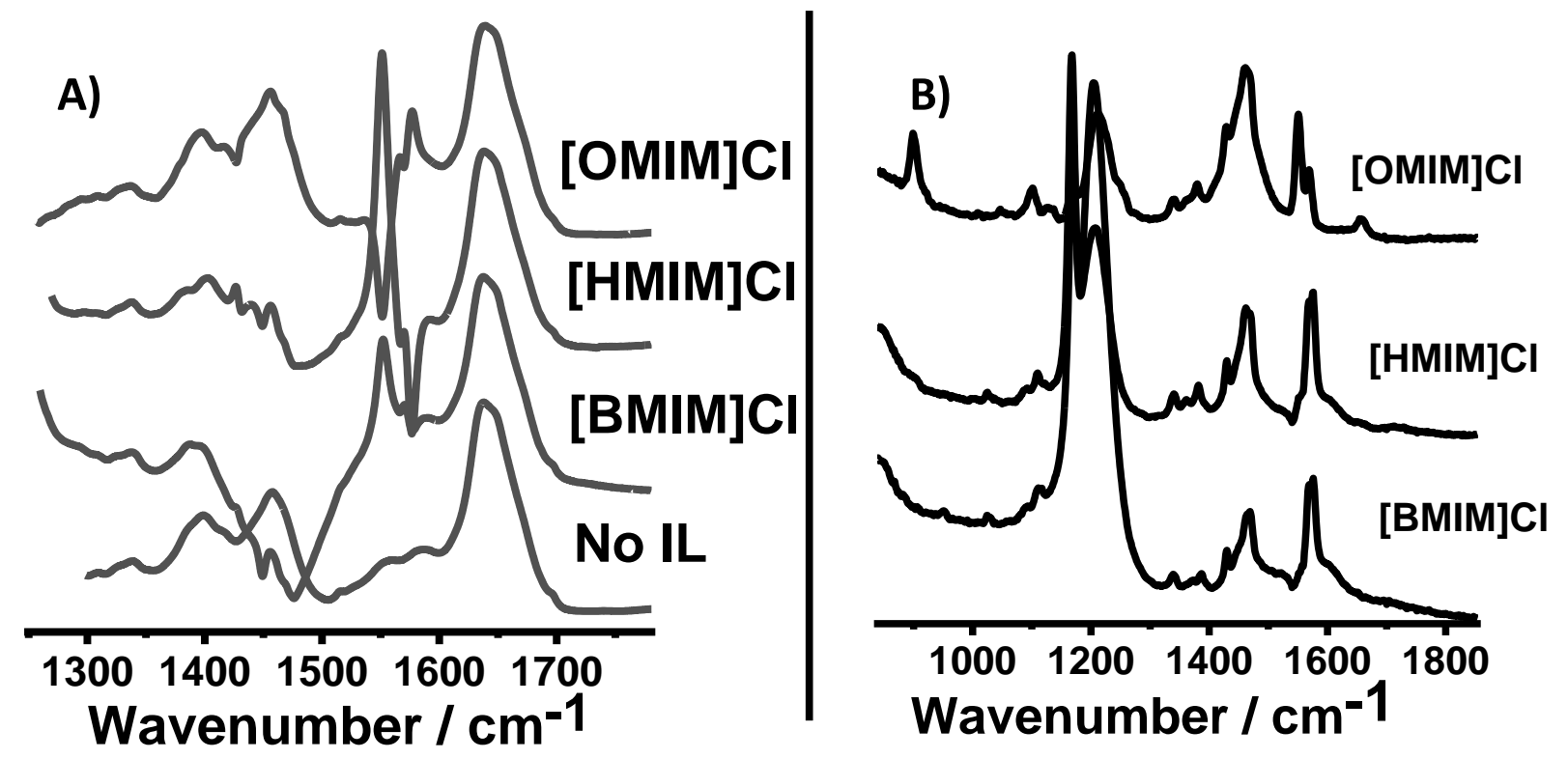

Figure S2. A) IR spectra for azurin in the presence of $1.0 \mathrm{M}$ ILs; B) IR spectra of $1.0 \mathrm{M}$ ILs in $\mathrm{D}_{2} \mathrm{O}$. 


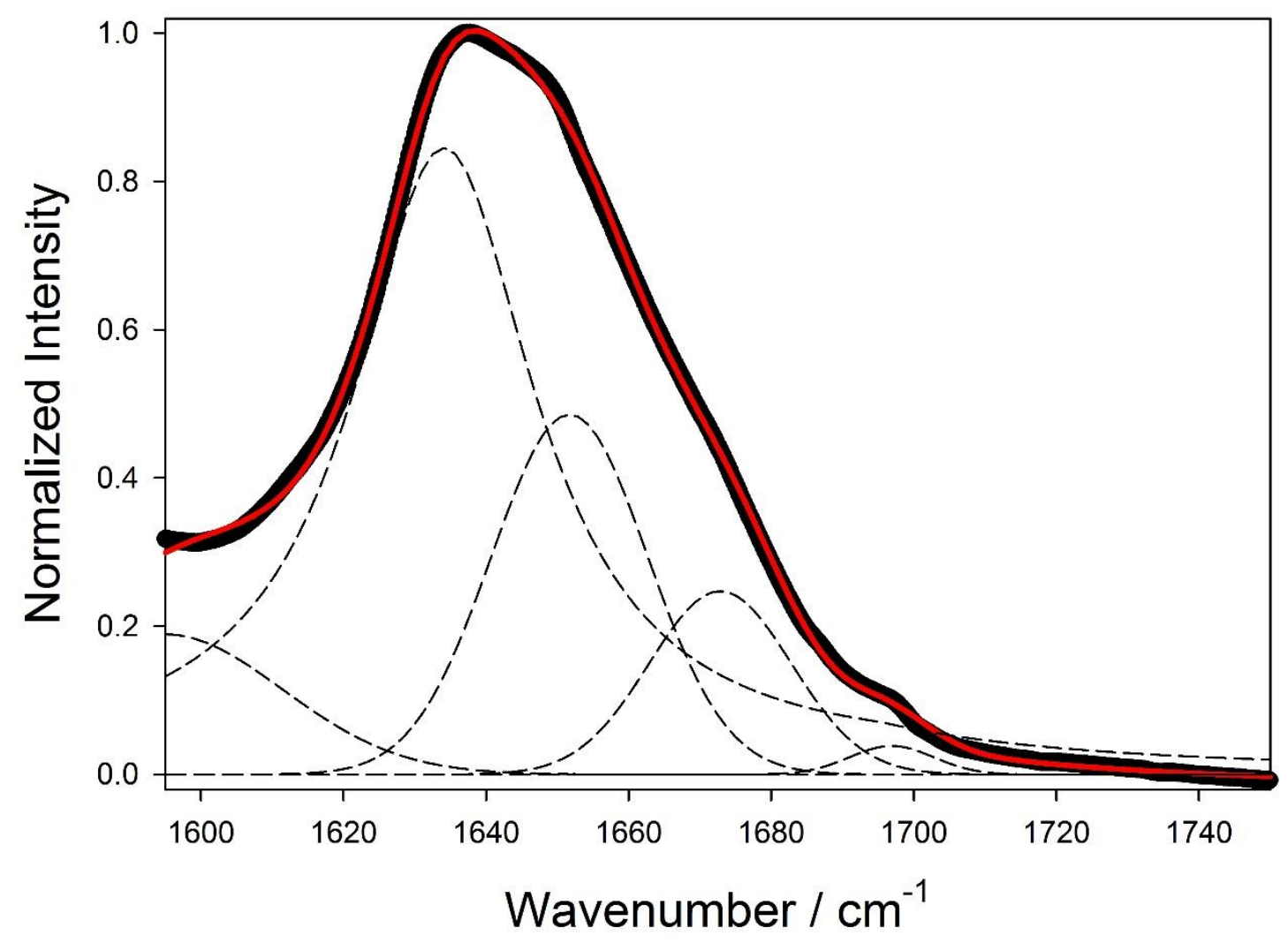

Figure S3. Example of an experimental amide l' band decomposed into sub-bands with Gaussian fits. Three amide I' bands are found: 1635 (Al1), 1652 (Al2) and $1673 \mathrm{~cm}^{-1}$ (Al3). 

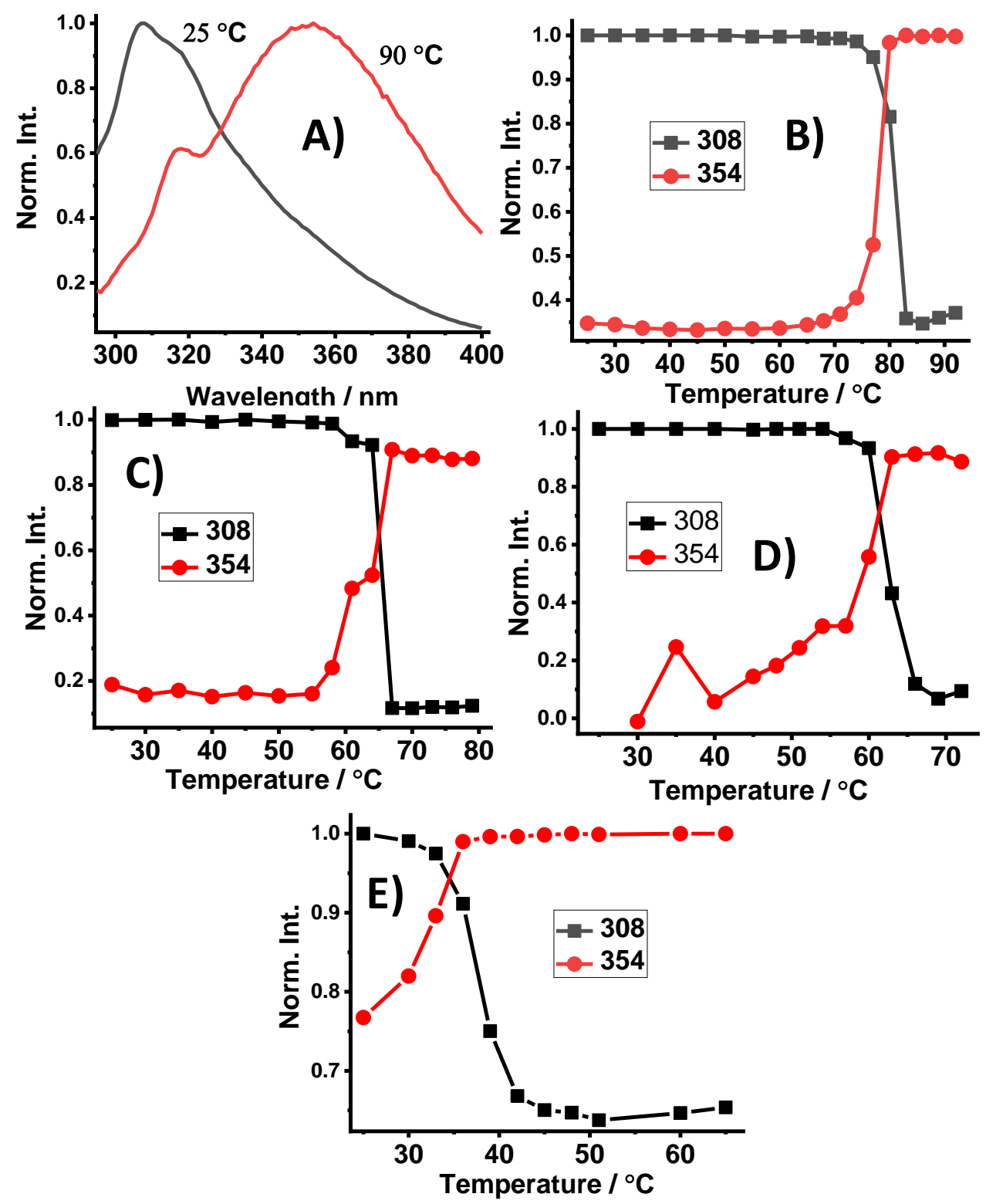

Figure S4. Fluorescence spectra of azurin in the presence of 1.0 M ILs. A) Fluorescence spectra of azurin (no IL) at low and high temperatures demonstrate that protein unfolding results in a shift of the fluorescence wavelength. B) The normalized intensities of the folded emission ( $308 \mathrm{~nm}$ ) and unfolded emission $(354 \mathrm{~nm}$ ) are shown vs. temperature for azurin with no IL, and the data show that azurin unfolds at $82^{\circ} \mathrm{C}$. C) Azurin unfolding with $1.0 \mathrm{M}[\mathrm{BMIM}] \mathrm{Cl}$, showing unfolding at $65^{\circ} \mathrm{C}$. D) Azurin unfolding with $1.0 \mathrm{M}[\mathrm{HMIM}] \mathrm{Cl}$, showing unfolding at $60^{\circ} \mathrm{C}$. (E) Azurin unfolding with $1.0 \mathrm{M}[\mathrm{OMIM}] \mathrm{Cl}$, showing unfolding at $38^{\circ} \mathrm{C}$. 

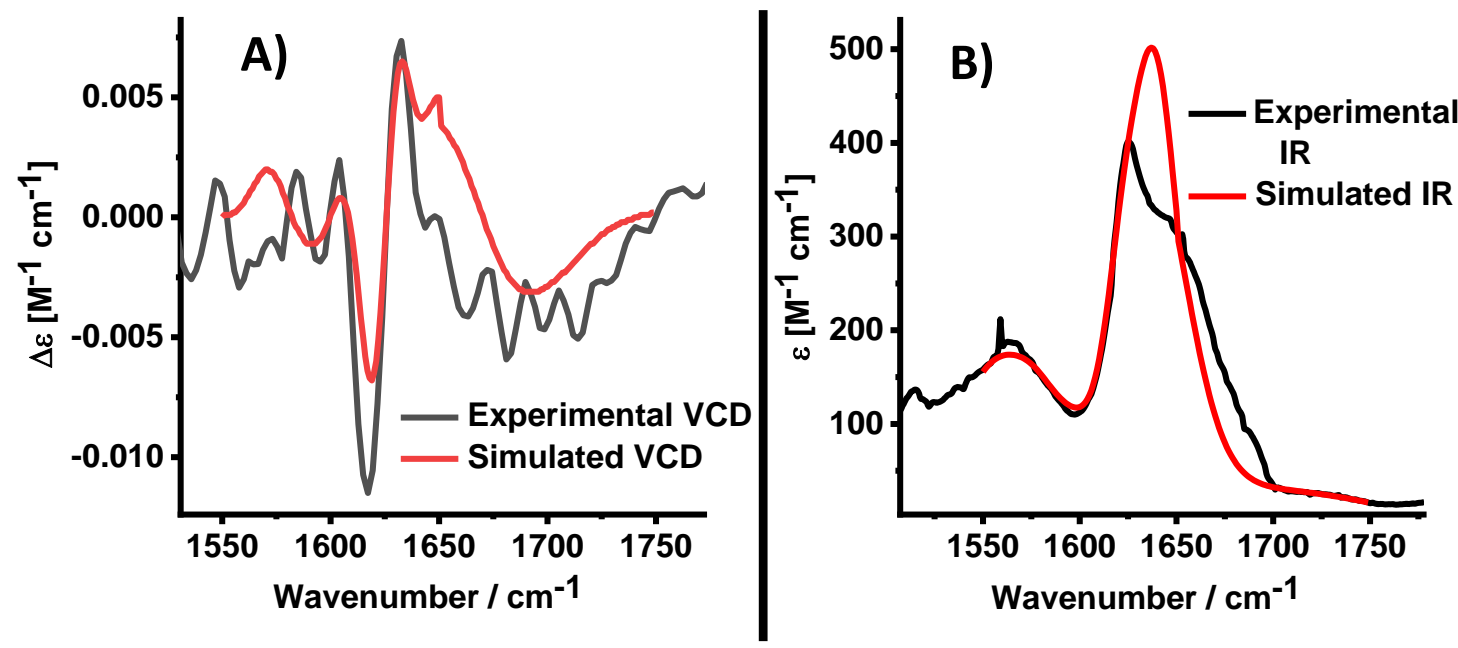

Figure S5. Experimental and simulated spectra of mCherry. A) VCD; B) FTIR. 


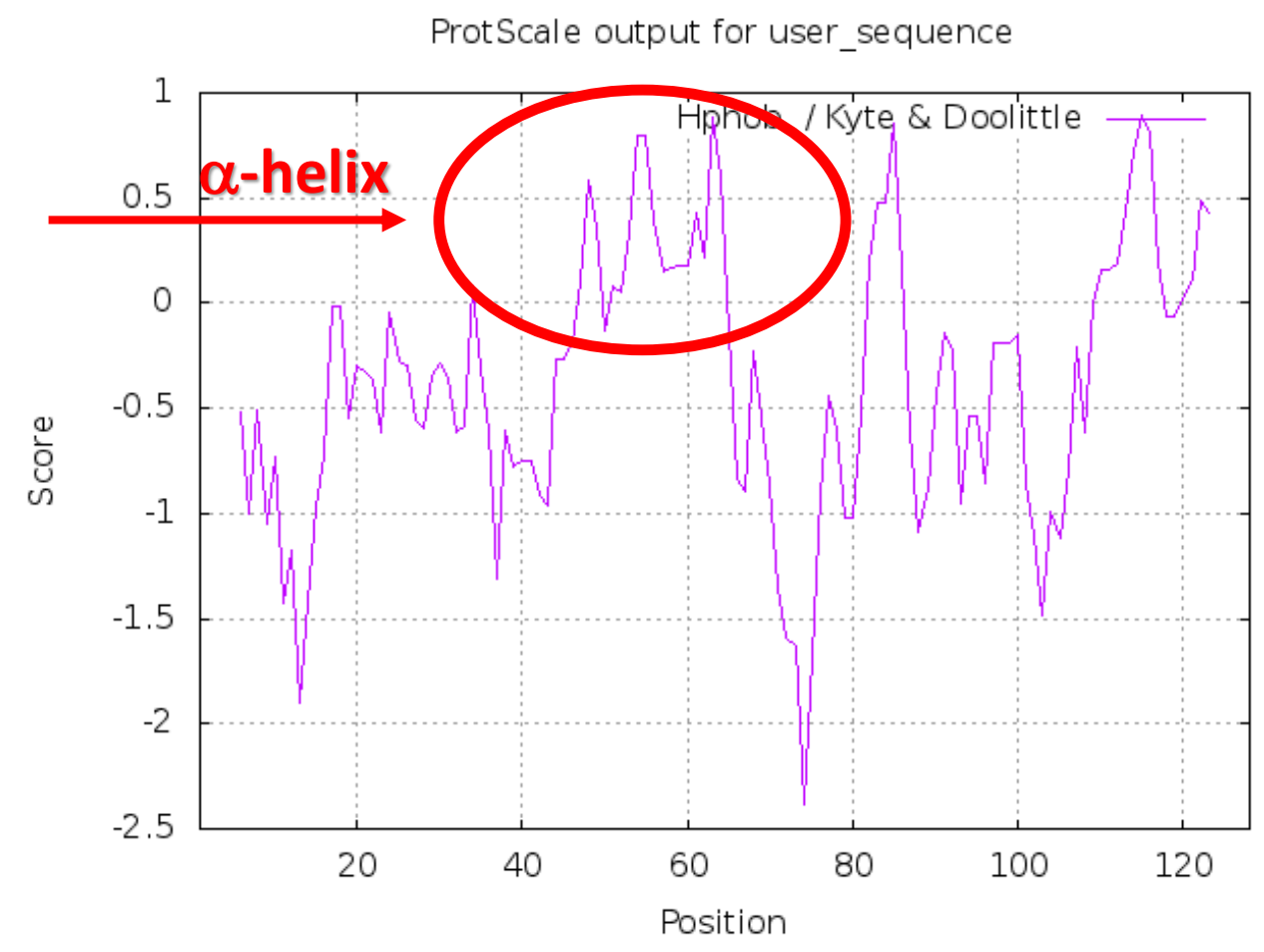

Figure S6. Analysis of the $1 \mathrm{AZU}$ pdb structure using the Kyte-Doolittle hydrophobicity scale, generated with the Expasy ProtScale online tool (https://web.expasy.org/protscale/), showing that the helical domain is mostly hydrophobic. 